\title{
EAPS8-1072: CALCANEUS OSTEITIS OF THE CHILD, A RARE PEDIATRIC CASE OF OSTEOARTICULAR TUBERCULOSIS
}

Maroua Ammous ${ }^{1}$, Faiza Safi ${ }^{1}$, Mohamed Ben Jemaa², Wajdi Bouaziz ${ }^{2}$, Zoubayer Ellouze ${ }^{2}$, Lamia Gargouri', Abdelmajid Mahfoudh'1

1: Pediatric Emergency Department, Hedi Chaker University Hospital of Sfax, Tunisia

2: Orthopedic Surgery Department, Habib Bourguiba University Hospital of Sfax, Tunisia

\section{Introduction:}

Bone and joint tuberculosis (BJT) represents $3-5 \%$ of all tuberculous lesions and approximately $15 \%$ of extra-pulmonary tuberculosis. The BJT is dominated by spinal locations that account for half of BJT cases. Peripheral BJT represents about 1 to $5 \%$ of all cases of tuberculosis . Rare and unusual locations of the BJT often pose a problem of diagnostic because of their atypical and non-specific clinical and radiological aspect.

Observation:

We present the case of a child aged 22 months, who consulted for left heel pain with functional impairment evolving for 9 months ago in a context of apyrexia. He was living in rural area, from economically deprived family. He received $\mathrm{BCG}$ vaccine and no contact patient with tuberculosis was identified. On admission, the child was eutrophic with conserved general state. The physical examination noted a swelling of the heel. On the other hand, there is no sign of other lesion. Tuberculin skin test was positive measuring $13 \mathrm{~mm}$.

Standard laboratory tests were normal. Standard radiography of the left ankle showed an osteolytic lesion invading all of the calcaneus with sharp contours of sclerosis (figure $\mathrm{n}^{\circ} 1$ ).

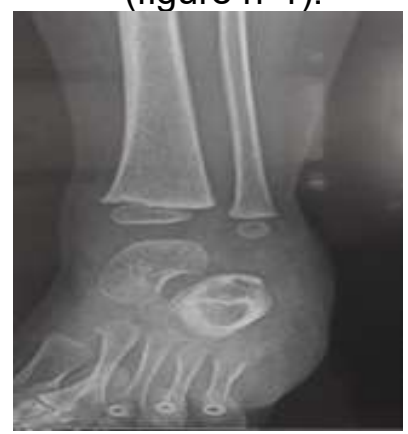

Figure $n^{\circ}$ : Standard radiography of the left ankle Osteolysis of the calcaneus surrounded by peripheral osteocondensation
Calcaneus tuberculosis was
evoked. Standard radiograph of the chest was normal. Mycobacterium tuberculosis wasn't recovered from spits and urines samples.

Surgery was decided. It consisted in abscess excision, washing and

biopsy of bone lesion.

Cytological study of drained liquid was hematic and rich in altered

leucocytes but culture was negative.

Histological examination of pathological samples found giant cell granuloma centered by caseous necrosis providing then the certainty of tuberculous origin.

Anti-tubercular treatment combining isoniazid, rifampicin, pyrazinamide and streptomycin was initiated for 2

months then it was followed by associating isoniazid and rifampicin for 9 months.

The ankle was immobilized by a plaster splint at the beginning of treatment.

Treatment adherence and tolerance was good. Favorable evolution was noted with regression of pain and skin healing. After one year of follow-up, the child walk without lameness and Radiological control of the ankle noted a good bone reconstruction and growth of the calcaneus (figure $n^{\circ} 2$ ).

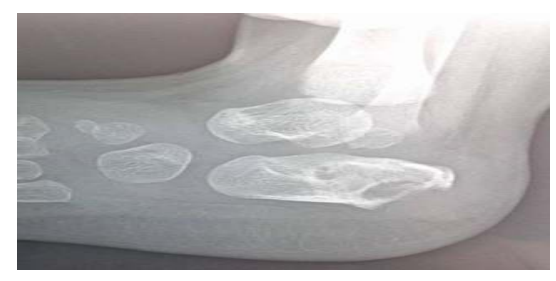

Figure $n^{\circ} 2$ : Standard Radiography of the ankle after treatment

Regression of the osteolysis and good bon growth of the calcaneus

\section{Discussion:}

Tuberculosis remains a major
In his bone and joint locations, peripheral tuberculosis is usually monofocal with osteomyelitis in 35 $\%$ of cases.

If tuberculous osteomyelitis of long bones metaphysis is the most common, the affection of the flat and small bones of the hand and feet is

rare. In the foot, the calcaneus location is the most common . At the early stage, radiological aspect is often normal.

The lesions reported are non-

specific with osteolysis and peripheral sclerosis.. In children, lesions are less sclerotic. We can

observe an acceleration of the ossification of the epiphyseal nuclei

and especially an aspect of migration through the physe of the metaphyseal lesions considered as a highly evocative aspect of BJT of the child. BJT management of the child is especially medical based on

anti-tuberculous therapy. It is

necessary to associate surgical treatment. It includes a drainage of osteo-articular lesions, a reduction

of dislocation, arthrodesis if

extensive joint destruction, corrective osteotomy if deformation, external fixation, curettage of a bone lesion, abscess drainage or removal of sequester. In children, the cure is complete with clinical and radiological recovery ad-integrum in case of early treatment. The sequelae are the prerogative of a diagnostic delay.

\section{Conclusion:}

Tuberculosis of children is still common.Its Clinical and radiological features are non-specific when location is rare posing several differential diagnoses. Bone and joint tuberculosis should be considered facing to any osteoarticular pain and swelling of the children with insidious evolution 